\title{
First mitral valve replacement using Edwards MITRIS RESILIA mitral valve in a patient with functional mitral regurgitation: a case report
}

Takashi Kakuta ${ }^{1}$, Tomoyuki Fujita ${ }^{1}$, Satoshi Kainuma ${ }^{1}$, Naonori Kawamoto ${ }^{1}$, Naoki Tadokoro $^{1}$, Ayumi Koga ${ }^{1}$, and Satsuki Fukushima ${ }^{1}$

${ }^{1}$ National Cerebral and Cardiovascular Center

June 3, 2021

\begin{abstract}
RESILIA tissue expected to prevent structural valve deterioration owing to calcification on leaflets was firstly mounted in a mitral bioprosthesis, named MITRIS RESILIA mitral valve (MITRIS). MITRIS has softer cuff than Magna Mitral Ease and stent posts that deflect to prevent suture looping. A 77-year-old man, having a history of long-standing atrial fibrillation, presented with dyspnea on effort. An echocardiography suggested atrial functional MR. We successfully replaced mitral valve using a 27-mm MITRIS, and his postoperative course was uneventful with good hemodynamics. MITRIS may have advantages for implantability and durability, making it a promising prosthesis for patients requiring mitral surgery.
\end{abstract}

First mitral valve replacement using Edwards MITRIS RESILIA mitral valve in a patient with functional mitral regurgitation: a case report

Takashi Kakuta, MD, Tomoyuki Fujita, MD, PhD, Satoshi Kainuma, MD, PhD, Naonori Kawamoto, MD, PhD, Naoki Tadokoro, MD, Ayumi Koga, MD, PhD, Satsuki Fukushima, MD, PhD

Department of Cardiovascular Surgery, National Cerebral and Cardiovascular Center, 5-7-1, Fujishirodai, Suita, Osaka, 565-8565, Japan

Short running title: MVR with MITRIS RESILIA

Funding: No funding was obtained for this report.

Conflict of Interest: none declared

Presentation : none presented

Corresponding author:

Tomoyuki Fujita, MD, PhD

Department of Cardiovascular Surgery, National Cerebral and Cardiovascular Center, 6-1, Kishibeshimmachi, Suita, Osaka, 564-8565, Japan.

Tel: 81-6-6170-1070

E-mail: tfujita@ncvc.go.jp

Abstract 
RESILIA tissue expected to prevent structural valve deterioration owing to calcification on leaflets was firstly mounted in a mitral bioprosthesis, named MITRIS RESILIA mitral valve (MITRIS). MITRIS has softer cuff than Magna Mitral Ease and stent posts that deflect to prevent suture looping. A 77-year-old man, having a history of long-standing atrial fibrillation, presented with dyspnea on effort. An echocardiography suggested atrial functional MR. We successfully replaced mitral valve using a 27-mm MITRIS, and his postoperative course was uneventful with good hemodynamics. MITRIS may have advantages for implantability and durability, making it a promising prosthesis for patients requiring mitral surgery.

\section{Keywords}

Mitral valve replacement, Functional mitral regurgitation, RESILIA tissue

\section{Introduction}

RESILIA tissue (Edwards Lifesciences, Irvine, CA) is a newly developed technology characterized by a stable capping process which permanently blocks residual aldehyde groups that bind to calcium. This processing helps prevent structural valve deterioration (SVD) due to calcification of leaflets [1]. MITRIS RESILIA mitral valve (MITRIS) (Fig. 1), which comprises RESILIA tissue mounted in a mitral bioprosthesis, is expected to contribute to long-term freedom from calcified SVD in patients who undergo mitral valve replacement (MVR). MITRIS also has softer cuffs than Edwards Magna Mitral Ease and stent posts that deflect to prevent suture looping, which may improve safety and implantability. Here we report the case of a patient with severe functional mitral regurgitation (MR) who underwent successful MVR using MITRIS as a first-inman. Data collection, analyses, and reporting for patients undergoing surgery in our institute were approved by our Institutional Review Board (Reference number: M30-026-5, on 24 ${ }^{\text {th }}$ March 2021). Written consent for submission and publication of this case report, including image and associated text, was obtained from the patient.

\section{Case Report}

A 77-year-old man (height: $161 \mathrm{~cm}$, weight: $60 \mathrm{~kg}$ ) with a long-history (more than 10 years) of atrial fibrillation $(\mathrm{AF})$ presented with dyspnea on effort related to congestive cardiac failure. Chest X-ray results showed cardiomegaly and pulmonary congestion with a cardio-thoracic ratio of $60 \%$. The electrocardiogram demonstrated AF. Transthoracic and transesophageal echocardiography (TTE, TEE) results revealed severe MR with shortening of the posterior leaflet which also had a small prolapse and enlarged mitral annulus. Doppler echocardiography revealed partially eccentric but mainly central severe MR jet diagnosed functional MR (Fig. 2A). The inter-commissure diameter and anterior-posterior diameter were 41 and $39 \mathrm{~mm}$, respectively. The left atrium was severely dilated with a left atrial volume of $196 \mathrm{ml}$, without any thrombus. The left ventricular diastolic/systolic dimension and ejection fraction were $58 / 39 \mathrm{~mm}$ and $65 \%$, respectively. The tricuspid regurgitation was moderately severe with a tricuspid diameter of $39 \mathrm{~mm}$ and a pressure gradient of $41 \mathrm{mmHg}$. Left ventriculogram showed Sellers III MR, and coronary angiogram revealed $75 \%$ stenosis in the left anterior descending artery (LAD).

The patient requested MVR rather than mitral valve repair after a conversation regarding durability of repair for functional MR.

Under general anesthesia, after a sternotomy, the left internal thoracic artery (LITA) was taken down and then cardiopulmonary bypass was started. Cardiac arrest was induced, and the left atrium was opened through right side left atriotomy. LITA was anastomosed to LAD and left atrial appendage was closed using $40 \mathrm{~mm}$ AtriClip (AtriCure, OH).

The anterior mitral leaflet was removed, and posterior leaflet was preserved (Supplemental Video). A total of 14 pledgeted everting mattress sutures were placed around the mitral annulus. The 27-mm sizer for MITRIS was fitted to the mitral annulus; therefore, a 27-mm MITRIS was picked up from a dry storage package and stent posts were deflected by rotating the attached dial (Fig. 2B), and all sutures were passed through the cuff of MITRIS, followed by placing it in intra-annular mitral position. The handle was removed, and all sutures were tied down. Finally, the holder keeping stent posts deflected was removed by cutting 
a suture (Fig. 2C). Subsequently, tricuspid annuloplasty was performed, and the cross clamp was released. Cardiac arrest time was $84 \mathrm{~min}$ and cardiopulmonary bypass time was $117 \mathrm{~min}$. Postoperative TTE showed that trans-mitral mean pressure gradient was $2.2 \mathrm{mmHg}$ with good left ventricular function (Fig. 2D), and regurgitation was absent. His postoperative course was uneventful.

\section{Comment}

This is the first report of MVR for mitral valve disease using MITRIS. RESILIA tissue used in MITRIS has been proven to reduce tissue calcification significantly in a randomized control study with juvenile sheep [1]. Mid-term outcomes of RESILIA tissue have been studied in two clinical trials which included 679 and 133 patients. Both studies presented no SVD up to 4 or 5 years as well as good hemodynamics $[2,3]$. The primary mode of failure of bovine bioprosthetic valves is SVD due to leaflet calcification, which contributes to hemodynamic deterioration, resulting in the need for re-MVR or transcatheter valve-in-valve procedures [4].

Whereas it is still controversial whether bovine or porcine leaflets protect prosthesis from SVD further, MITRIS may provide even higher protection from SVD as predicted based on the results of aortic bioprosthesis with RESILIA tissue [2,3,5]. Consequently, MVR using MITRIS becomes a favorable option for patients who require mitral surgery, especially in younger age.

\section{Acknowledgment}

We thank Edwards Lifesciences for providing the image of the study valve and the valve technical characteristics.

\section{References}

1. Flameng W, Hermans H, Verbeken E, Meuris B. A randomized assessment of an advanced tissue preservation technology in the juvenile sheep model. J Thorac Cardiovasc Surg. 2015;149: 340-5.

2. Johnston DR, Griffith BP, Puskas JD, Bavaria JE, Svensson LG, COMMENCE trial Investigators. $\mathrm{J}$ Intermediate-term outcomes of aortic valve replacement using a bioprosthesis with a novel tissue. Thorac Cardiovasc Surg . 2020:S0022-5223(20)30474-8. doi: 10.1016/j.jtcvs.2020.01.095. Online ahead of print.

3. Bartus K, Litwinowicz R, Bilewska A, et al. Final 5-year outcomes following aortic valve replacement with a RESILIA tissue bioprosthesis. Eur J Cardiothorac Surg . 2021;59:434-41.

4. Rodriguez-Gabella T, Voisine P, Puri R, Pibarot P, Rodés-Cabau J. Aortic bioprosthetic valve durability incidence, mechanisms, predictors, and management of surgical and transcatheter valve degeneration. J Am Coll Cardiol . 2017;70:1013-28.

5. Malvindi PG, Mastro F, Kowalewski M, et al. Durability of mitral valve bioprostheses: a meta-analysis of long-term follow-up studies. Ann Thorac Surg . 2020;109:603-11.

\section{Figure and video legends}

Figure 1: MITRIS RESILIA mitral valve

Figure 2: (A) Preoperative doppler echocardiography revealed central severe MR jet. (B) Stent posts of MITRIS RESILIA Mitral Valve were deflected to prevent suture looping by rotating the attached dial. (C) MITRIS RESILIA mitral valve was placed in intra-annular mitral position. (D) Postoperative echocardiography revealed transmitral mean pressure gradient was $2.2 \mathrm{mmHg}$ with good left ventricular function.

Supplemental Video: Mitral valve replacement using $27 \mathrm{~mm}$ MITRIS RESILIS mitral valve in a patient with functional mitral regurgitation. 


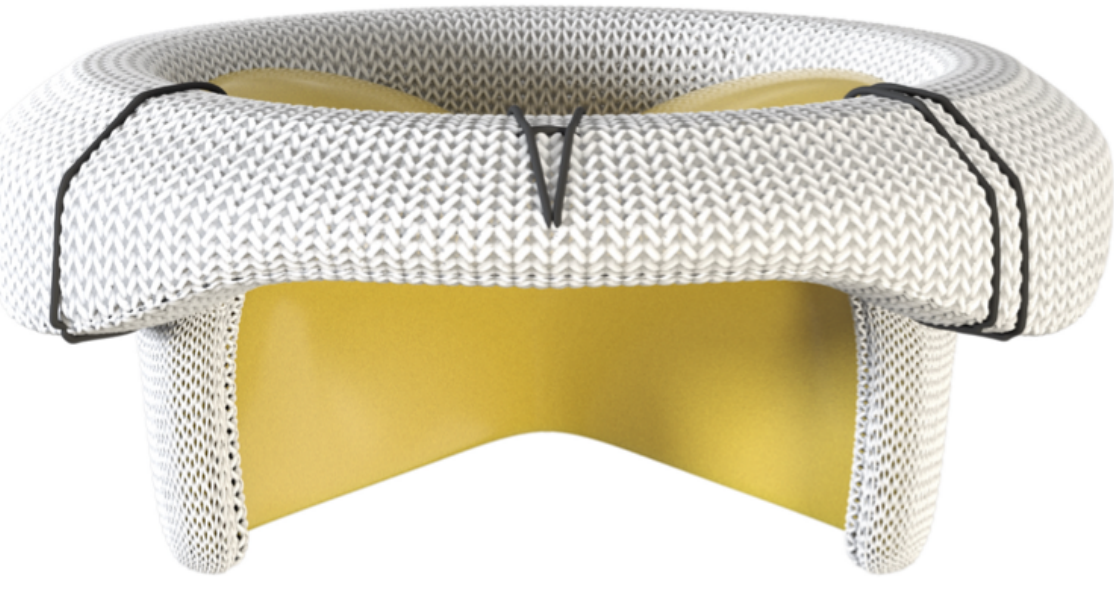

A

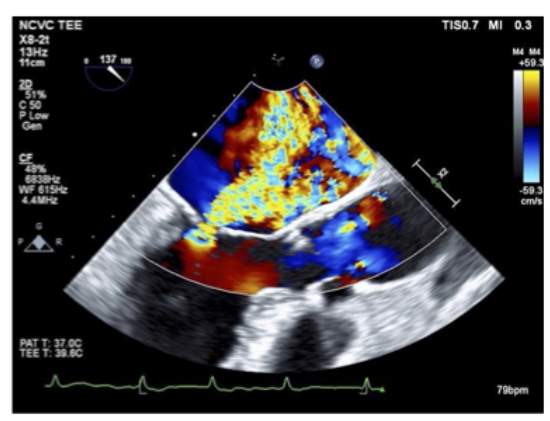

C

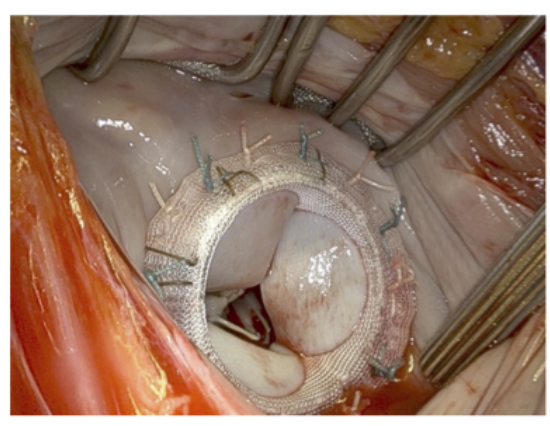

B

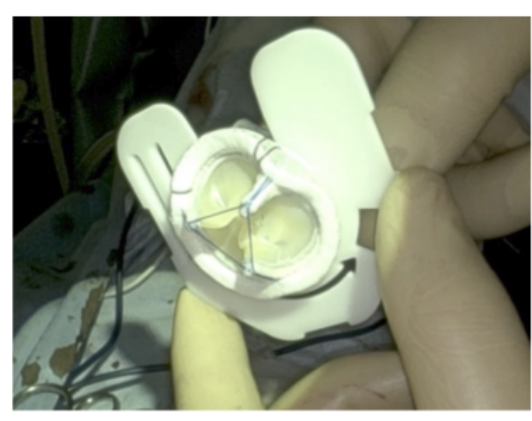

D

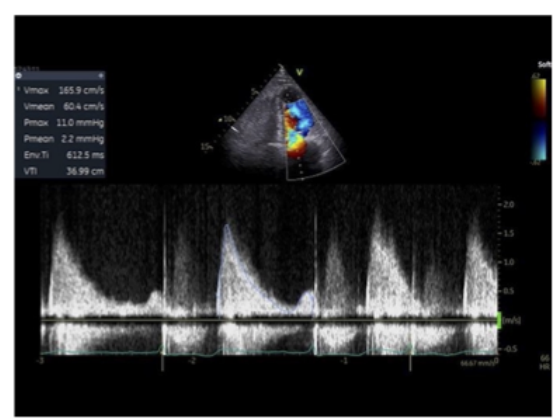

